Trauma Berufskrankh 2004 · 6 [Suppl 4] : S479-S483 DOI 10.1007/s10039-003-0800-6

Online publiziert: 10. Januar 2004

(c) Springer-Verlag 2004

M.Schuldt ${ }^{1} \cdot$ W. Kaduk ${ }^{2}$

${ }^{1}$ Klinik für Kinderchirurgie im Zentrum für Kinder- und Jugendmedizin, Ernst-Moritz-Arndt-Universität, Greifswald

${ }^{2}$ Klinik für Mund-, Kiefer- und Gesichtschirurgie der Ernst-Moritz-Arndt-Universität, Greifswald

\title{
Differenzierter Lasereinsatz
} zur Narbenbehandlung

\section{Laserarten}

In den vergangenen Jahren hat sich die Lasertherapie in der Behandlung von Narben etabliert. Die ersten Versuche der Narben- und Keloidtherapie mittels Laser wurden mit Dauerstrichlasern unternommen und zeigten hohe Rezidivraten und unzureichende Resultate. Erst mittels moderner gepulster Lasersysteme wurden bei richtiger Indikationsstellung gute Erfolge erzielt. Das Ziel der Behandlung besteht in der graduellen Verbesserung des kosmetischen Bildes und auch der Funktion. Der Patient sollte sich nicht auf eine vollständige Befreiung von der Narbe einstellen. Heute gibt es eine Vielzahl von Lasersystemen, deren Einsatzgebiet mit dem Fortschreiten der medizinischen Forschung zunehmend ausgeweitet wird. In der Narbenbehandlung werden gewebsabtragende Laser benutzt, wie der $\mathrm{CO}_{2}$-und der Erbium:YAG-Laser, und gefäßzerstörende Laser, zu denen der Farbstofflaser und der Neodym:YAG-Laser gerechnet werden.

\section{Biologische Wirkung des Lasers im Gewebe}

Die Wellenlänge der Laserstrahlung entscheidet darüber, in welchen Strukturen der Haut die Laserenergie absorbiert wird. Die Nutzung von Absorptionsmaxima der Hautpigmente erlaubt eine selektive Therapie der Hautstrukturen, in denen diese
Pigmente enthalten sind. Das emittierte Licht des $\mathrm{CO}_{2}$-Lasers und des Erbium:YAG-Lasers hat einen hohen Absorptionskoeffizienten für Wasser und ist damit für das oberflächliche Vaporisieren der Haut geeignet. Für den Farbstofflaser und den Neodym:YAG-Laser liegt das Absorptionsmaximum im Hämoglobin. Sie werden vornehmlich zum selektiven Zerstören von Gefäßen [5, 13] eingesetzt.

\section{Farbstofflaser}

Der blitzlampengepumpte, gepulste Farbstofflaser ist ein Flüssigkeitslaser, dessen Licht in sehr kurzen Applikationsimpulsen über ein Fokussierhandstück in die Haut geschossen wird. Der Wirkungsmechanismus des Farbstofflasers im Bereich der gefäßreichen Komponente der Narbe liegt in der hohen Absorption des emittierten Lichtes durch feine Kapillargefäße, die das Substrat der persistierenden Rötung der Narbe darstellen. Die hohe Absorption des Lichtes durch das Hämoglobin führt durch den sehr schnellen Energieeintrag in die Gefäße zu deren Zerreißung. Durch die den Wärmeleiteigenschaften des Gewebes angepassten Expositionszeiten und die sehr hohe Spezifität für Gefäße kommt es zu keiner thermischen Schädigung des umgebenden Gewebes. Die Wirksamkeit des Lasers auf die hypertrophe Komponente der Narbe wird durch die Gewebehypoxie nach selektiver Photothermolyse der Kapillargefäße erklärt. Dies führt zu blasse Narben, die sich in einem ruhenden Stadium befinden. 


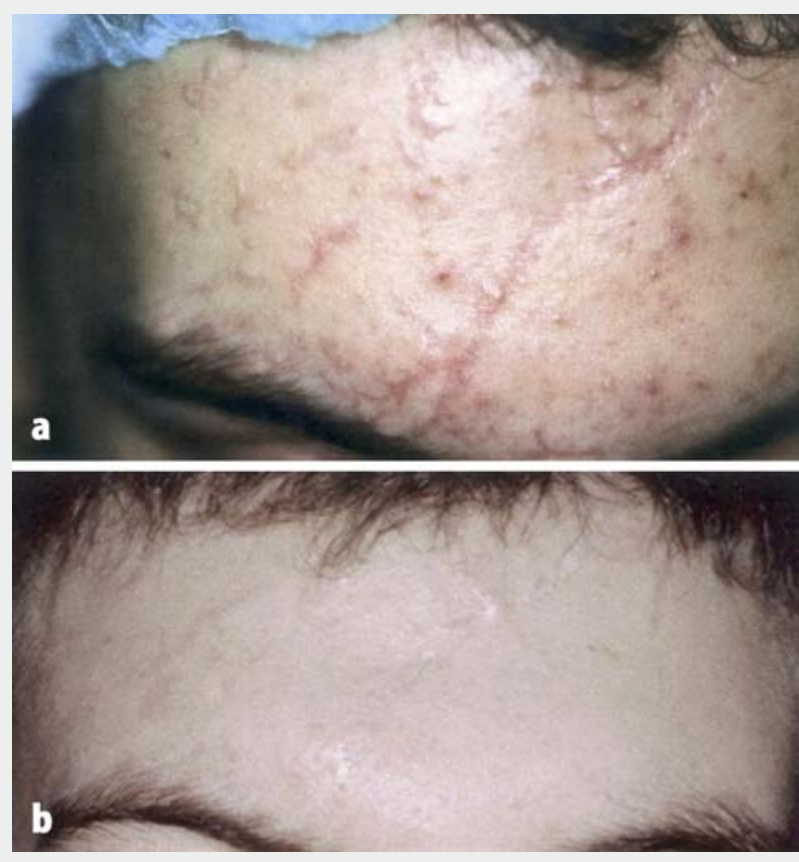

Abb. $1<$ a Erythematöse Narben nach Glassplitterverletzungen im Stirnbereich. b Resultat nach 2-maliger Farbstofflaserbehandlung im Abstand von 6 Wochen
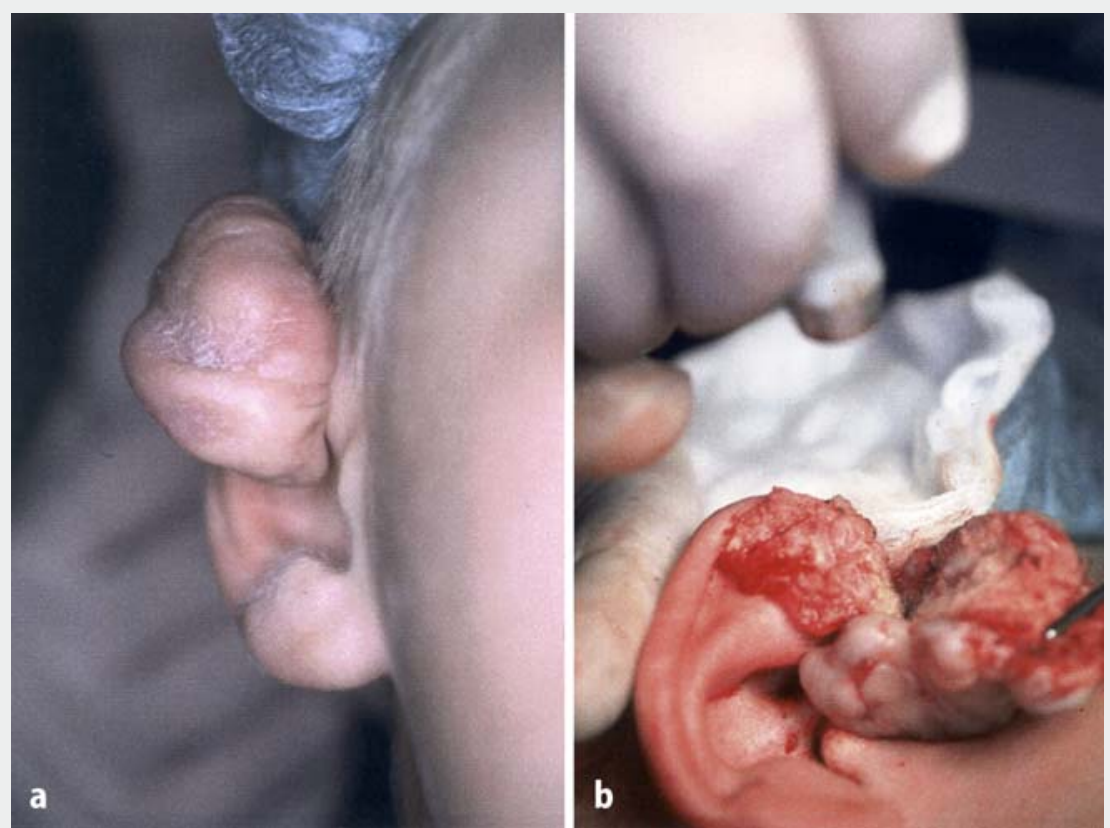

Abb. $2 \Delta$ a Ausgeprägtes, exophytisches Keloid mit Proliferationstendenz nach plastischer Korrektur an der Ohrmuschel. b Resektion des Keloides mit dem Neodym:YAG-Laser in der Schneidfunktion zur groben Gewebereduktion

einem Abbau von Kollagen und hemmt gleichzeitig die erneute Narbenproliferation $[1,2,3,11]$. Der Farbstofflaser ist geeignet zur Behandlung frischer, gut vaskularisierter Narben $(\bullet$ Abb. 1a,b). Bei älteren und blassen Narben wirkt der Farbstofflaser weniger effektiv.

\section{Neodym:YAG-Laser}

Der Neodym: YAG-Laser ist ein Festkörperlaser, dessen im nahen Infrarotbereich gelegenes Licht über Fokussierhandstücke oder einen einfachen dünnen Lichtleiter zum Gewebe gelangt. Er besitzt gleichfalls eine hohe Absorption im Blut. Durch lange Expositionszeiten kommt es zu einer relativ gleichmäßigen Verteilung des Lichtes im Gewebe. Gerade in der Schneidfunktion entsteht dadurch ein guter Hämostaseeffekt, den man sich beim Versiegeln von Schnittflächen bei der Massenreduktion von gut durchbluteten exophytischen Keloiden zu Nutze macht. Für einen präzisen Gewebeabtrag ist er nicht geeignet $([10,11] ; \bullet$ Abb. 2a,b).

\section{$\mathrm{CO}_{2}$-Laser}

Der $\mathrm{CO}_{2}$-Laser zählt zu den Gaslasern. Das im Infrarotbereich gelegene Licht des Lasers wird mittels Fokussierhandstücken oder programmierbaren Scannern ins Gewebe gebracht. Er hat sein Absorptionsmaximum im wasserhaltigen Gewebe. Dadurch kommt es beim Applizieren auf die Haut zu einer unspezifischen Aufnahme der Energie in den obersten Hautschichten. Folge ist ein schnelles Erhitzen und Verdampfen von intra- und extrazellulärem Wasser bei Temperaturen von über $100^{\circ} \mathrm{C}$ unter weitgehender Schonung der umliegenden Hautpartien.

Mit dem gepulsten $\mathrm{CO}_{2}$-Laser ist damit ein präzises, sequenzielles Abtragen feinster Hautschichten möglich. Kleine Nervenendigungen, Lymphgefäße und Blutgefäße bis zu einem Durchmesser von 0,5 $\mathrm{mm}$ werden photokoaguliert, wodurch ein relativ blutarmes Arbeiten ermöglicht wird sowie postoperativ geringere Schmerzen und Ödeme auftreten. Durch die vorhandene thermische Komponente kommt es unmittelbar während der Behandlung zum sog. Kollagen-Shrinking, einem sichtbaren Schrumpfen der Narbe. Verursacht wird dies durch eine hitzebedingte anhaltende Kontraktion der Kollagenfibrillen mit Verkürzung auf $1 / 3$ der Faserlänge. Dieser Shrinkingeffekt trägt zu einer späteren Kollageninduktion bei $[6,11,12]$.

Eingesetzt wird der gepulste $\mathrm{CO}_{2}$-Laser zur Behandlung atrophischer Narben. Mit dem Fokussierhandstück wird zunächst eine Nivellierung der randständigen Hautstufen vorgenommen und anschließend zum Glätten großflächig Gewebe mit dem Scanner abgetragen. Die so entfernte Schichtdicke der Haut liegt pro Durchgang bei ca. 50-100 $\mu \mathrm{m}$. Auch bei hypertrophen Narben und Keloiden ist der $\mathrm{CO}_{2}-$ Laser zu verwenden. Mit dem Fokussier- 
handstück können Keloide im CuttingMode reseziert und hypertrophe Narben nivelliert werden (• Abb. 3,4).

Das vollständige, einzeitige Abtragen proliferierender, hypertropher Narben mit $\operatorname{dem} \mathrm{CO}_{2}$-Laser lehnen wir wegen der $\mathrm{Ge}$ fahr eines Rezidivs ab. Wir führen in diesen Fällen mehrere Sitzungen mit zwischenzeitlichen Pausenintervallen von 68 Wochen durch - eine Zeitspanne, die der Regenerationsphase der Haut entspricht. Gute Erfahrungen haben wir mit der Kombinationsbehandlung von gepulstem $\mathrm{CO}_{2}$-Laser zur Ablation und zum Shrinking mit sofort nachfolgender und wenn nötig wiederholter Farbstofflaserbehandlung zur Unterbindung einer erneuten Proliferation gemacht.

\section{Erbium:YAG-Laser}

Der Erbium:YAG-Laser ist ein Festkörperlaser, der Licht im mittleren Infrarotbereich emittiert. Auch bei diesem ist das Arbeiten mit Fokussierhandstücken oder einem computergesteuerten Scanner möglich. Wie beim $\mathrm{CO}_{2}$-Laser ist auch hier das Ziel des Laserstrahls das Wasser in den Zellen. Der Erbium:YAG-Laser besitzt jedoch im Vergleich zum $\mathrm{CO}_{2}$-Laser eine 15-mal stärkere Absorption für Wasser. Dadurch ist es möglich, ihn für ein noch nebenwirkungsfreieres Abtragen feinster Hautschichten einzusetzen. Es kommt bei extrem schnellen Temperaturanstiegen auf mehr als $100^{\circ} \mathrm{C}$ mit der Folge einer explosionsartigen Hautabtragung zur sog. kalten oder athermischen Ablation. Eine Blutstillung wird mit dem Erbium:YAGLaser dadurch nicht erreicht. Der Laser ist deutlich schmerzärmer als der $\mathrm{CO}_{2}$ Laser und kann auch ohne Anästhesieverfahren eingesetzt werden. Wegen der nur minimalen thermischen Wirkung bleibt auch der Shrinking-Effekt auf das Narbengewebe aus.

Um mit dem Erbium:YAG-Laser neben dem Gewebeabtrag auch einen Straffungseffekt zu erreichen, bieten Laser der neueren Generation einen sog. thermischen Mode an. Durch Änderung der Leistungsparameter mit längerer Impulsdauer und geringerer Energiedichte wird subablativ eine Aufwärmung der oberflächlichen Hautschichten [4, 7, 8, 9] erzeugt. Einsatzgebiet des Erbium:YAG-Lasers sind

Trauma Berufskrankh 2004 - 6 [Suppl 4] : S479-S483

DOI 10.1007/s10039-003-0800-6

C) Springer-Verlag 2004

M. Schuldt $\cdot$ W. Kaduk

\section{Differenzierter Lasereinsatz zur Narbenbehandlung}

\section{Zusammenfassung}

In den vergangenen Jahren haben sich Abtragungslaser, wie der Erbium:YAG- und der $\mathrm{CO}_{2}$-Laser sowie der Farbstoff- und der Neodym:YAGLaser als gefäßzerstörende Laser in der oft schwierigen Behandlung von Narben etabliert.

Spezielle Einsatzgebiete sind atrophische und hypertrophe Narben und Keloide. Die Anwendungsmöglichkeiten hängen in erster Linie von der Wellenlänge des emittierten Laserlichtes und der damit verbundenen biologischen Wirkung im Gewebe ab. Dementsprechend hat jedes Lasersystem sein spezielles Indikationsgebiet wie Reduktion des Narbengewebes oder Verhinde-

rung einer Proliferation. In vielen Fällen ist eine Laserkombinationsbehandlung erforderlich. Eine intersive Nachbehandlung nach Lasertherapie ist zur Sicherung des postoperativen Ergebnisses erforderlich. Unsere Ergebnisse zeigen eindrucksvoll, dass die heute zur Verfügung stehenden Lasersysteme eine erfolgversprechende Ergänzung zu den etablierten konservativen und operativen Verfahren in der Narbenbehandlung darstellen.

\section{Schlüsselwörter}

Laser $\cdot$ Narben · Keloide

\section{Differentiated laser application in treatment of scars}

\section{Abstract}

In the past few years skin-ablative laser systems such as erbium:YAG laser and carbon dioxide laser and vascular destroying laser systems such as dye laser and neodymium:YAG laser have established themselves in the difficult treatment of scars. Special fields of application are atrophic and hypertrophic scars as well as keloids. Applications depend on wavelength of the emitted laser light and the associated biological effect in the tissue. Accordingly, different types of laser systems are used for special indications such as reduction of scar tissue or of proliferation. In many cases a combined laser treatment is required. Intensive supplementary therapy is essential to protect the good postoperative result. Our results clearly demonstrate that the laser systems available to date are a promising supplementation to the already established conservative and operative procedures in the treatment of striking scars.

\section{Keywords}

Laser $\cdot$ Scars $\cdot$ Keloids 

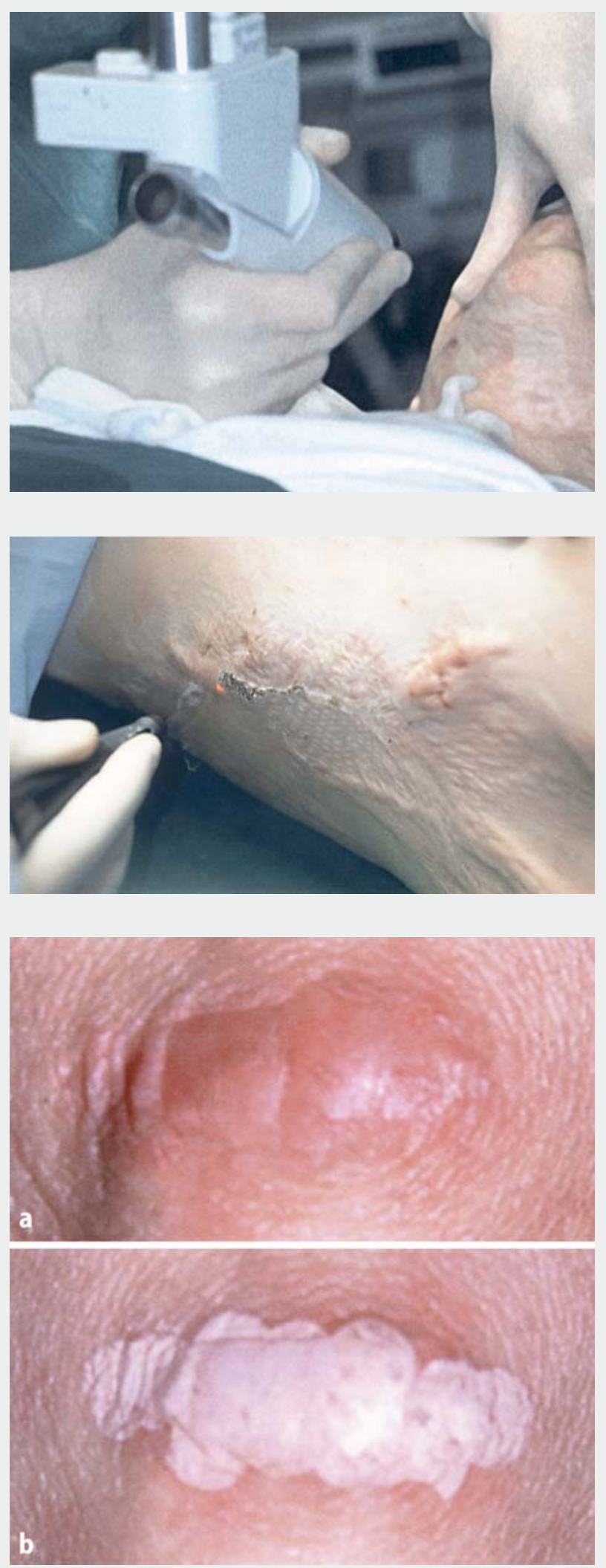

Abb. $3<$ Großflächige Ablation mit dem $\mathrm{CO}_{2}-$ Laser mit Scanner zur Narbennivellierung im Halsbereich

Abb. $4<$ Grobes Glätten einer Verbrennungsnarbe am Thorax mit dem $\mathrm{CO}_{2}$-Laser mit einem Fokussierhandstück

Abb. $5<$ a Hypertrophe Narbe im Ellbogenbereich. b Weißlicher Gewebeabtrag im Narbenbereich nach feinschichtiger, präziser Ablation mit dem Erbium:YAG-Laser mit einem Fokussierhandstück - Spotgröße $5 \mathrm{~mm}$ blasse hypertrophe und atrophische Narben. Zum Schneiden und Resezieren ist der Erbium-Laser nicht geeignet (• Abb. $5 a, b)$.

\section{Nachbehandlung}

Um den durch die Lasertherapie erzielten Erfolg auch postoperativ zu halten, sind zusätzliche Therapiemaßnahmen erforderlich. Entscheidend ist das Unterbinden bzw. Minimieren erneuter Narbenwucherung. Unmittelbar nach Laserbehandlung sollten blande, rückfettende Salben wie Vasilinum album eingesetzt werden. Daneben können Narbenexterna, die antiphlogistische und durchblutungsfördernde Wirkstoffe enthalten, eingesetzt werden. Spezielle lokale Dermatika, wie Contractubex, hemmen das Wachstum von Fibroblasten, führen zu einer Auflockerung der Kollagenstrukturen und fördern die Wasserbindung im Narbengewebe. Bei Anzeichen einer erneuten Proliferation kann das Narbengewebe mit Steroiden, wie beispielsweise Triamzinolon, unterspritzt werden.

Mit Farbstofflaserbehandlungen nimmt man dem Narbenwachstum durch Reduktion des Vaskularisationsgrades die Grundlage. Auch eine konsequente und langanhaltende Kompressionsbehandlung, v. a. bei ausgedehnten Verbrennungsnarben, beugt einer erneuten $\mathrm{Wu}$ cherung vor.

\section{Komplikationen}

Nach der Behandlung mit Ablationslasern können verzögerte Wundheilungen, Infektionen oder auch Blutungen auftreten. Auch bei oberflächlicher Abtragung können sich temporäre oder anhaltende Pigmentstörungen sowie Erytheme ausbilden. Speziell beim Farbstofflaser sind eine ausgeprägte Purpura oder auch Verbrennungen 2. Grades möglich. Als Hauptkomplikation ist mittel- oder langfristig mit Rezidiven zu rechnen.

\section{Fazit}

Die heute zur Verfügung stehenden Lasersysteme bieten bei sorgfältiger Indikationsstellung, richtiger Patientenauswahl und entsprechender Erfahrung des behandelnden 
Arztes eine zusätzliche Therapiemöglichkeit in der schwierigen Behandlung von Narben und Keloiden. Für geeignete Fälle stellen die genannten Laser eine gute und erfolgversprechende Ergänzung zu den etablierten konservativen und operativen Narbenbehandlungsverfahren dar.

\section{Korrespondierender Autor}

\section{Dr. M. Schuldt}

Klinik für Kinderchirurgie im Zentrum für Kinder- und Jugendmedizin der Ernst-Moritz-Arndt-Universität Greifswald, Sauerbruchstraße 1, 17487 Greifswald E-mail:

matthias.schuldt@kinderchirurgie.uni-greifswald.de

Interessenkonflikt: Der korrespondierende Autor versichert, dass keine Verbindungen mit einer Firma, deren Produkt in dem Artikel genannt ist, oder einer Firma, die ein Konkurrenzprodukt vertreibt, bestehen.

\section{Literatur}

1. Alster TS (1994) Improvement of erythematous and hypertrophic scars by the 585 -nm flashlamp-pumped pulsed dye laser. Ann Plast Surg 32: 186-190

2. Connell PG, Harland CC (2000) Treatment of keloid scars with pulsed dye laser and intralesional steroid.J Cutan Laser Ther 2: 147-150

3. Dörschel K, Helfmann J (2003) Dye lasers. In: Berlien HP, Müller GJ (eds) Applied laser medicine.Springer, Berlin Heidelberg New York Tokio, p 37-40

4. Frank F, Wondrazek F (2003) Erbium:YAG Laser. In: Berlien HP, Müller GJ (eds) Applied laser medicine. Springer, Berlin Heidelberg New York Tokio, p 45-49

5. Fuchs B, Berlien HP, Philipp CM (2003) The laser's position in medicine. In: Berlien HP, Müller GJ (eds) Applied laser medicine. Springer, Berlin Heidelberg New York Tokio, p 187-197

6. Glotz M (2003) $\mathrm{CO}_{2}$ Lasers. In: Berlien HP, Müller GJ (Hrsg) Applied laser medicine.Springer, Berlin Heidelberg New York, p 33-36

7. Kaufmann R, Beier C (2001) Erbium:YAG laser therapy of skin lesions. Med Laser Appl 16: 252-263

8. Kunzi-Rapp K, Diebolder R, Wortmann S et al. (2001) Treatment of hypertrophic scars and keloids on the bases of thermal induced collagen reactions by a new erbium:YAG-Laser.J Cut Laser Therapy 3: 32-33

9. Majaron B, Srinivas SM, Huang H, Nelson S (2000) Deep coagulation of dermal collagen with repetitive Er:YAG laser irradiation. Lasers Surg Med 26: 215-222

10. Schaldach B (2003) Nd:YAG lasers. In: Berlien HP, Müller GJ (eds) Applied laser medicine. Springer, Berlin Heidelberg New York Tokio, p 23-25

11. Scharschmidt $D$ (2003) Laser treatment of scars and keloids. In: Berlien HP, Müller GJ (eds) Applied laser medicine.Springer, Berlin Heidelberg New York Tokio, p 545-554

12. Scharschmidt D, Algermissen B, Philipp C, Berlien HP (1998) Prinzipien der Laserbehandlung von Narben und Keloiden.J DGPW 16: 7-9

13. Steiner $R$ (2003) Interactions of laser radiation with biological tissue. In: Berlien HP, Müller GJ (eds) Applied laser medicine.Springer, Berlin Heidelberg New York Tokio, p 101-106 